\title{
Flood Disaster Risk Analysis for Songhua River Basin Based on Theory of Information Diffusion
}

\author{
Chang Yi, Chongfu Huang, and Yaozhong Pan \\ College of Resources Science and Technology, Beijing Normal University, \\ 100875 Beijing, China \\ yichang531@ires.cn
}

\begin{abstract}
Flood disaster risk analysis, the essential of which is to estimate the probability distribution of flood disaster loss, is an important nonstructural measure for flood control and disaster reduction. In the paper, a frequency analysis method of flood disaster loss based on fuzzy mathematics theory of information diffusion with short time series of flood disaster samples is suggested for flood disaster risk analysis. With the support of historic disaster data, the method has been applied to flood disaster risk analysis for Songhua River Basin and turned out to be effective and practical.
\end{abstract}

Keywords: Information Diffusion, Flood Disaster, Risk Analysis, Songhua River Basin.

\section{Introduction}

In recent years, flood disaster loss has been sharply increasing with the rapid socialeconomic development. More and more attention has been paid to flood disaster risk analysis, an important nonstructural measure of flood prevention and disaster reduction ${ }^{[1,2]}$. Predominating flood disaster risk is an important basis for decisions on flood control and flood insurance. When the historic flood data is sufficient, the results of risk evaluation can be obtained with considerable satisfaction generally by probability and statistics. However, in fact, the phenomenon of lack of experimental flood data occurs frequently, resulting in fewer samples that can only provide us with finite and incomplete knowledge to understand risk. In this case, it is inappropriate to take risk analysis by means of probability and statistics with a small sample, the sample size of which is usually less than 30 , as the great fuzziness and uncertainty of the activity can sometimes lead to very unstable results that may even depart quite far from the real situation. Actually, one sample in a small sample set can not be treated as a specific observation due to its fuzziness and uncertainty, but a representation of the samples or a set-value sample as well as an observation sample of a fuzzy set. In this paper, fuzzy mathematics theory of information diffusion is used to establish the flood risk evaluation model with a small number of experimental samples. The model is then applied to flood risk analysis for Songhua River Basin in North-East China. 


\section{Definition of Flood Risk}

The definition of Flood Risk is the basis of study on flood risk. The majority of researchers believe that risk is the occurrence probability of adverse events and the seriousness of their possible aftereffects. Flood disaster denotes the disaster situation that forms when the hazard, flood, acts on disaster-affected bodies in certain disasterformative environment. Based on the definition of risk mentioned above, it can be deduced that adverse event of flood risk is flood, and the aftereffect is flood loss, namely disaster situation. Therefore, flood risk refers to the occurrence probability of flood at different intensity and the flood loss possibly caused, which is actually threefold: (1) flood, measurement of characteristics and magnitude of the flood, such as the highest water level, the flood peak discharge, the grade of flood volume in interval and the condition for flood happening, etc; (2) probability, the occurrence probability of flood event mainly denotes the occurrence frequency and the recurrence interval of those flood events that exceed certain grade or numerical value; (3) loss, the loss possibly caused by the occurrence of flood event basically includes flood disaster economic loss, personnel casualties, environment pollution, social influence, etc. Flood risk analysis is just to study on the probability distribution of flood loss (or of disaster situation).

\section{Flood Disaster Risk Analysis Based on Information Diffusion}

Flood risk analysis utilizing historic disaster data mostly adopts the method of flood disaster loss frequency analysis. Flood frequency analysis has got widely application in hydrology, but fewer frequency analyses have been done on flood disaster loss (disaster situation). Most scholars believe that flood disaster loss (disaster situation) data fall short of the requirement of frequency analysis for data consistency. Indeed, with respect to flood disaster economic loss (currency measurement), its gradual increase with the development of society leads to the non-conformity to the requirement for data consistency, however, certain disaster indicators like disasteraffected rate of arable land, in certain period, can be considered as a random variable whose frequency can be analyzed on.

In the statistical risk based on probability and statistics, the uncertainty involved in risk is relevant to the similitude degree to some standard mode, which can be depicted by fuzzy sets membership in mathematics theory. Information diffusion is just a fuzzy mathematic set-value method for samples, considering optimizing the use of fuzzy information of samples in order to offset the information deficiency. The method can turn an observed sample into a fuzzy set, that is, turn a single point sample into a setvalue sample ${ }^{[3]}$. The simplest model of information diffusion is normal diffusion model.

Suppose $X$ is the natural disaster indicator, and the transcendental frequency distribution of $X$ in $T$ years can be defined as disaster risk. Concretely, $X$ is given as economic loss indicator which consists of some specific quantitative values, noted as 
$X=\left\{x_{1}, x_{2}, \ldots, x_{n}\right\}$. And suppose the probability for loss transcending $X_{i}$ is $p_{i}\left(x \geq x_{i}\right)$, $\mathrm{i}=1,2,3, \ldots, \mathrm{n}$, then the probability distribution is $P=\left\{p_{1}, p_{2}, \ldots, p_{n}\right\}$, which can be named as loss risk caused by disaster, or disaster risk.

If the actual records of the natural disaster indicator in some region during the past $n$ years are $y_{1}, y_{2}, \ldots, y_{n}$ respectively, then the observed sample set is $Y=\left\{y_{1}, y_{2}, \ldots, y_{m}\right\}$, in which, $y_{j},(\mathrm{j}=1,2, \ldots, \mathrm{m})$ is named as disaster sample.

Suppose that the universe discourse of disaster indicator is $V=\left\{v_{1}, v_{2}, \ldots, v_{n}\right\}$. Usually, the natural disaster indicator $X$ is taken as the universe discourse $V$, and each $x$ in $X$ is taken as corresponding element in the universe discourse $V$.

A single point observed sample $y$ can diffuse the information it takes to every $v_{i}$ according to $R\left(v_{i}\right), R\left(v_{i}\right)=\frac{1}{\eta \sqrt{2 \pi}} \exp \left[-\frac{\left(y-v_{i}\right)^{2}}{2 \eta^{2}}\right]$, in which $\eta$ is the diffusion coefficient. $\eta=\alpha(b-a) /(m-1)$ can be typically determined by the maximum value - $\mathrm{b}$, the minimum value - a, and the number of samples - $\mathrm{m}$, of the sample set, in which $\alpha$ is the parameter relevant to the sample size as shown in equation (1).

$$
\eta=\left\{\begin{array}{l}
1.6987(b-a) /(m-1) \ldots \ldots \ldots \ldots . .1<m \leq 5 \\
1.43416(b-a) /(m-1) \ldots \ldots \ldots . .5 \leq m \leq 10 \\
1.4208(b-a) /(m-1) \ldots \ldots \ldots \ldots \ldots . . .10 \leq m
\end{array} .\right.
$$

Thus, the single point sample y has been turned into the fuzzy set $y^{*}$ which takes $\mu_{y}\left(v_{i}\right)$ as its membership function.

During risk evaluation, in order to maintain the status of each set-value sample the same, every sample $y_{j}$ should be diffused according to equation $R\left(v_{i}\right)$, that is,

$$
R_{j}\left(v_{i}\right)=\frac{1}{\eta \sqrt{2 \pi}} \exp \left[-\frac{\left(y_{j}-v_{i}\right)^{2}}{2 \eta^{2}}\right] .
$$

The membership function of corresponding fuzzy sets is

$$
\mu_{y_{j}}\left(v_{i}\right)=\frac{R_{j}\left(v_{i}\right)}{\sum_{i=1}^{n} R_{j}\left(v_{i}\right)} .
$$

The $\mu_{y_{j}}\left(v_{i}\right)$ is called as the normalized information distribution of sample $y_{j}$.

Suppose that the number of samples, whose observation value is $v_{i}$, deduced by information diffusion is

$$
l\left(v_{i}\right)=\sum_{j=1}^{m} \mu_{y_{j}}\left(v_{i}\right)
$$


Then, the frequency value of a sample falling at $v_{i}$ is

$$
P\left(v_{i}\right)=\frac{l\left(v_{i}\right)}{\sum_{i=1}^{n} l\left(v_{i}\right)} .
$$

The frequency value can be taken as the estimation value for probability. Apparently, the probability value of transcending $v_{i}$ should be

$$
p\left(v_{i}\right)=\sum_{k=i}^{n} p\left(v_{k}\right) .
$$

The $p\left(v_{i}\right)$ is the very risk estimation value needed to be calculated.

\section{Flood Disaster Risk Analysis-Taking Songhua River Basin for Example}

Songhua River is the largest branch river of Heilongjiang Basin in China. After the confluence of the upper reaches-Nen River and the second Songhua River-at Sanchahe, Fuyu County, Jilin Province, the main stream of Songhua River runs to meet Heilong River at Tongjiang County. In history, Songhua River had repeatedly suffered from attacks of flood. During the 240 years from 1746 to 1985,104 times of flood disasters had happened in Songhua River Basin altogether, among which 15 were extraordinary ones ${ }^{[4]}$.

To analyze the spatial characteristics of flood risk in Songhua River Basin, and according to the historic disaster data having been collected so far, the paper divides Songhua River Basin into three sub-basins respectively, that is, Nen River sub-basin, the main stream of Songhua River sub-basin and the second Songhua River sub-basin. Afterwards, in terms of the different provinces these sub-basins belong to, Nen River sub-basin is divided further into Heilonjiang district, Neimenggu district and Jilin district; the main stream of Songhua River sub-basin is divided further into Heilongjiang district and Jilin district; and the Second Songhua River sub-basin mainly lies in Jilin Province.

Disaster-affected rate has been chosen as the disaster indicator in flood risk analysis. In a certain time period, disaster-affected rate commonly has quite fine consistency. Disaster-affected rate means the ratio of disaster-affected area to crop area. In practical calculation, it is so difficult to collect the crop area in every region year by year that the arable land area is taken as a substitute. Then, calculate the disaster-affected rate ${ }^{1}$ of each district in Songhua River Basin for the 25 years from 1965 to 1989 year by year, the result of which is shown in Table 1.

\footnotetext{
${ }^{1}$ The disaster-affected area data was provided by Songhua River and Liaohe River Conservancy Commission, and the arable land area data was acquired by measuring from the electronic 1:4,000,000 land use map.
} 
Table 1. The disaster-affected rate of each district in Songhua River Basin year by year

\begin{tabular}{ccccccc}
\hline \multirow{2}{*}{ Dist } & \multicolumn{2}{c}{$\begin{array}{c}\text { The main stream of } \\
\text { Songhua River }\end{array}$} & $\begin{array}{c}\text { The second } \\
\text { Songhua River }\end{array}$ & Nen River & \\
\cline { 2 - 7 } Year & Jilin & Heilongjiang & Jilin & Jilin & Heilongjiang & Neimenggu \\
\hline 1965 & 2.259077769 & 1.015268 & 0.925307 & 1.381932 & 0.691327 & 1.787675 \\
1966 & 0 & 0.411851 & 0.11615 & 0.028548 & 0.141697 & 0.079912 \\
1967 & 0 & 0.048061 & 0.04391 & 0 & 0.171606 & 0 \\
1968 & 0 & 0.057183 & 0.030985 & 0 & 0.500109 & 0 \\
1969 & 0.219576086 & 0.267162 & 0.174934 & 0.071613 & 2.404771 & 2.345648 \\
1970 & 0 & 0.031505 & 0.495763 & 0 & 0.397145 & 0 \\
1971 & 0.75515585 & 0.189877 & 0.89804 & 0.604837 & 0.26934 & 1.802717 \\
1972 & 0 & 1.325423 & 0.145011 & 0 & 0.524133 & 0 \\
1973 & 0.305547675 & 1.530334 & 1.518452 & 0 & 0.262802 & 0.188028 \\
1974 & 0.066221359 & 0.999304 & 0.071886 & 0.181451 & 0.052299 & 0.06581 \\
1975 & 0.101074706 & 0.401715 & 1.209662 & 0.558869 & 0.346807 & 0.00282 \\
1976 & 0 & 0.093672 & 0 & 0 & 0.025332 & 0 \\
1977 & 0 & 0.881982 & 0.402276 & 0.140322 & 0.92945 & 2.749908 \\
1978 & 0 & 0.805626 & 0.057898 & 0 & 0.170952 & 0 \\
1979 & 0 & 0.313196 & 0.313039 & 0 & 0.193343 & 0.296144 \\
1980 & 0.644786918 & 0.431109 & 0.227166 & 0 & 1.773751 & 1.41491 \\
1981 & 5.088588648 & 7.406639 & 1.925509 & 1.075642 & 2.686695 & 1.354271 \\
1982 & 0.108626265 & 0.566168 & 0.110661 & 0 & 0.027457 & 0.288623 \\
1983 & 2.038920794 & 4.881731 & 1.905501 & 2.533541 & 3.354814 & 2.842982 \\
1984 & 1.067674194 & 4.369283 & 1.210725 & 1.694028 & 4.586944 & 2.101212 \\
1985 & 5.769390691 & 7.780818 & 9.782116 & 7.028206 & 2.58128 & 2.326375 \\
1986 & 9.726407343 & 3.819079 & 8.38247 & 12.41077 & 2.624753 & 6.26932 \\
1987 & 0.411269493 & 8.870246 & 2.185253 & 5.180308 & 1.505556 & 3.866324 \\
1988 & 4.173688292 & 10.65009 & 1.361047 & 6.365305 & 6.362329 & 10.74203 \\
1989 & 5.424342557 & 3.027896 & 2.323713 & 0 & 1.884396 & 5.739551 \\
\hline
\end{tabular}

Suppose that $S_{j}(j=1,2, \ldots 25)$ are the disaster-affected area in the period of the 25 years respectively, and $S_{j}{ }^{\prime}$ is the crop area of the corresponding year, then the disaster-affected rate can be expressed as

$$
I_{j}=\frac{S_{j}}{S_{j}^{\prime}} \times 100 \%(j=1,2, \ldots 25) .
$$

Based on equation (7), the disaster-affected rates of the 25 samples can be calculated out, that is, the observed sample set $I=\left\{I_{1}, I_{2}, \ldots, I_{25}\right\}$.In view of both convenience and accuracy of the computer-aided calculation, the universe discourse of disaster-affected rate is taken as $V=\left\{v_{1}, v_{2}, \ldots, v_{71}\right\}=\{0,0.002,0.004, \ldots, 0.140\}$. The normalized information distribution of each $I_{j}$, that is, $\mu_{I_{j}}\left(v_{i}\right)$, can be obtained according to equation (2) and (3). Then, based on equation (4), (5) and (6), the flood risk estimation value $P\left(v_{i}\right)$, that is, disaster-affected risk probability, of each district in Songhua River Basin can be worked out, as shown in table 2. 
Table 2. The flood disaster risk evaluation values of each district in Songhua River Basin

\begin{tabular}{|c|c|c|c|c|c|c|}
\hline \multirow[b]{2}{*}{$P\left(v_{i}\right)$} & \multicolumn{2}{|c|}{$\begin{array}{c}\text { The main stream of } \\
\text { Songhua River }\end{array}$} & \multicolumn{2}{|l|}{$\begin{array}{c}\text { The second } \\
\text { Songhua River }\end{array}$} & \multicolumn{2}{|l|}{ Nen River } \\
\hline & Jilin & Heilongjiang & Jilin & Jilin & Heilongjiang & Neimenggu \\
\hline 0.000 & 1.000000 & 1.000000 & 1.000000 & 1.000000 & 1.000000 & 1.000000 \\
\hline 0.002 & 0.860689 & 0.919120 & 0.893011 & 0.875254 & 0.883896 & 0.900566 \\
\hline 0.004 & 0.720842 & 0.829189 & 0.779280 & 0.750705 & 0.748927 & 0.801654 \\
\hline 0.006 & 0.594220 & 0.736548 & 0.668676 & 0.634099 & 0.622752 & 0.711202 \\
\hline 0.008 & 0.490093 & 0.647643 & 0.568698 & 0.531315 & 0.525449 & 0.634224 \\
\hline 0.010 & 0.411498 & 0.567664 & 0.482775 & 0.445509 & 0.460861 & 0.571848 \\
\hline 0.012 & 0.356112 & 0.499791 & 0.410570 & 0.377105 & 0.420676 & 0.521880 \\
\hline 0.014 & 0.318592 & 0.445143 & 0.349633 & 0.324440 & 0.392956 & 0.480364 \\
\hline 0.016 & 0.292944 & 0.403203 & 0.297178 & 0.284728 & 0.368197 & 0.443287 \\
\hline 0.018 & 0.274120 & 0.372388 & 0.251184 & 0.254942 & 0.341847 & 0.407742 \\
\hline 0.020 & 0.258722 & 0.350553 & 0.210706 & 0.232409 & 0.313480 & 0.372332 \\
\hline 0.022 & 0.245061 & 0.335359 & 0.175669 & 0.215067 & 0.283484 & 0.336989 \\
\hline 0.024 & 0.232767 & 0.324545 & 0.146439 & 0.201478 & 0.250857 & 0.302507 \\
\hline 0.026 & 0.222180 & 0.316142 & 0.123352 & 0.190709 & 0.215064 & 0.270046 \\
\hline 0.028 & 0.213715 & 0.308624 & 0.106343 & 0.182171 & 0.178988 & 0.240714 \\
\hline 0.030 & 0.207430 & 0.300966 & 0.094790 & 0.175483 & 0.147662 & 0.215284 \\
\hline 0.032 & 0.202903 & 0.292601 & 0.087622 & 0.170355 & 0.123933 & 0.194038 \\
\hline 0.034 & 0.199356 & 0.283297 & 0.083585 & 0.166518 & 0.107040 & 0.176776 \\
\hline 0.036 & 0.195902 & 0.273014 & 0.081532 & 0.163681 & 0.095094 & 0.162954 \\
\hline 0.038 & 0.191781 & 0.261811 & 0.080593 & 0.161519 & 0.086960 & 0.151906 \\
\hline 0.040 & 0.186512 & 0.249814 & 0.080207 & 0.159684 & 0.081591 & 0.143034 \\
\hline 0.042 & 0.179904 & 0.237233 & 0.080065 & 0.157829 & 0.077113 & 0.135899 \\
\hline 0.044 & 0.171945 & 0.224398 & 0.080018 & 0.155638 & 0.071438 & 0.130178 \\
\hline 0.046 & 0.162630 & 0.211760 & 0.080005 & 0.152860 & 0.063748 & 0.125551 \\
\hline 0.048 & 0.151853 & 0.199851 & 0.080001 & 0.149334 & 0.055211 & 0.121600 \\
\hline 0.050 & 0.139473 & 0.189203 & 0.080000 & 0.145004 & 0.047964 & 0.117781 \\
\hline 0.052 & 0.125521 & 0.180235 & 0.080000 & 0.139904 & 0.043312 & 0.113493 \\
\hline 0.054 & 0.110412 & 0.173169 & 0.080000 & 0.134124 & 0.041005 & 0.108206 \\
\hline 0.056 & 0.095000 & 0.167977 & 0.080000 & 0.127763 & 0.039875 & 0.101606 \\
\hline 0.058 & 0.080407 & 0.164400 & 0.080000 & 0.120891 & 0.038574 & 0.093700 \\
\hline 0.060 & 0.067706 & 0.162008 & 0.080000 & 0.113541 & 0.035762 & 0.084832 \\
\hline 0.062 & 0.057608 & 0.160269 & 0.079999 & 0.105740 & 0.030418 & 0.075610 \\
\hline 0.064 & 0.050308 & 0.158609 & 0.079994 & 0.097545 & 0.022670 & 0.066753 \\
\hline 0.066 & 0.045529 & 0.156440 & 0.079978 & 0.089097 & 0.014205 & 0.058911 \\
\hline 0.068 & 0.042704 & 0.153203 & 0.079930 & 0.080632 & 0.007245 & 0.052517 \\
\hline 0.070 & 0.041200 & 0.148439 & 0.079798 & 0.072464 & 0.002938 & 0.047724 \\
\hline 0.072 & 0.040482 & 0.141894 & 0.079479 & 0.064930 & 0.000932 & 0.044427 \\
\hline 0.074 & 0.040174 & 0.133616 & 0.078794 & 0.058326 & 0.000229 & 0.042348 \\
\hline 0.076 & 0.040055 & 0.123982 & 0.077486 & 0.052847 & 0.000043 & 0.041148 \\
\hline 0.078 & 0.040009 & 0.113608 & 0.075269 & 0.048561 & 0.000006 & 0.040517 \\
\hline 0.080 & 0.039976 & 0.103167 & 0.071931 & 0.045406 & 0.000001 & 0.040213 \\
\hline 0.082 & 0.039910 & 0.093190 & 0.067451 & 0.043226 & 0.000000 & 0.040080 \\
\hline 0.084 & 0.039745 & 0.083957 & 0.062075 & 0.041816 & 0.000000 & 0.040026 \\
\hline 0.086 & 0.039354 & 0.075528 & 0.056247 & 0.040961 & 0.000000 & 0.040001 \\
\hline 0.088 & 0.038536 & 0.067865 & 0.050425 & 0.040478 & 0.000000 & 0.039977 \\
\hline 0.090 & 0.037017 & 0.060954 & 0.044866 & 0.040223 & 0.000000 & 0.039928 \\
\hline 0.092 & 0.034516 & 0.054843 & 0.039531 & 0.040097 & 0.000000 & 0.039810 \\
\hline
\end{tabular}


Table 2. (Continued)

\begin{tabular}{lllllll}
\hline 0.094 & 0.030866 & 0.049580 & 0.034171 & 0.040039 & 0.000000 & 0.039544 \\
0.096 & 0.026146 & 0.045101 & 0.028561 & 0.040014 & 0.000000 & 0.039003 \\
0.098 & 0.020735 & 0.041166 & 0.022711 & 0.040001 & 0.000000 & 0.038002 \\
0.100 & 0.015237 & 0.037399 & 0.016927 & 0.039990 & 0.000000 & 0.036327 \\
0.102 & 0.010286 & 0.033404 & 0.011681 & 0.039968 & 0.000000 & 0.033786 \\
0.104 & 0.006334 & 0.028929 & 0.007393 & 0.039920 & 0.000000 & 0.030296 \\
0.106 & 0.003538 & 0.023975 & 0.004261 & 0.039816 & 0.000000 & 0.025953 \\
0.108 & 0.001785 & 0.018799 & 0.002224 & 0.039606 & 0.000000 & 0.021058 \\
0.110 & 0.000810 & 0.013818 & 0.001048 & 0.039209 & 0.000000 & 0.016060 \\
0.112 & 0.000330 & 0.009453 & 0.000444 & 0.038514 & 0.000000 & 0.011437 \\
0.114 & 0.000120 & 0.005985 & 0.000169 & 0.037385 & 0.000000 & 0.007565 \\
0.116 & 0.000039 & 0.003491 & 0.000057 & 0.035681 & 0.000000 & 0.004627 \\
0.118 & 0.000011 & 0.001870 & 0.000017 & 0.033293 & 0.000000 & 0.002607 \\
0.120 & 0.000003 & 0.000917 & 0.000005 & 0.030185 & 0.000000 & 0.001349 \\
0.122 & 0.000001 & 0.000411 & 0.000001 & 0.026430 & 0.000000 & 0.000640 \\
0.124 & 0.000000 & 0.000168 & 0.000000 & 0.022217 & 0.000000 & 0.000278 \\
0.126 & 0.000000 & 0.000062 & 0.000000 & 0.017828 & 0.000000 & 0.000110 \\
0.128 & 0.000000 & 0.000021 & 0.000000 & 0.013581 & 0.000000 & 0.000040 \\
0.130 & 0.000000 & 0.000007 & 0.000000 & 0.009765 & 0.000000 & 0.000013 \\
\hline
\end{tabular}

Table 2 has shown the general situation of overall risk level for each district that Heilongjiang district of the main stream of Songhua River and Jilin district of Nen River are the two districts suffering from the flood disaster risk most, while Heilongjiang district in Nen River endures the lowest level of risk. According to the risk evaluation results shown above, it can be summed up that the disaster-affected rates of each district at different probability levels are as follows in table 3:

Table 3. The disaster-affected rates of each district in Songhua River Basin at different probability levels

\begin{tabular}{rcccccc}
\hline Dist & \multicolumn{2}{c}{$\begin{array}{c}\text { The main stream of } \\
\text { Songhua River }\end{array}$} & \multicolumn{2}{c}{$\begin{array}{c}\text { The second } \\
\text { Songhua River }\end{array}$} & \multicolumn{3}{c}{ Nen River } \\
\cline { 2 - 7 } Probability & Jilin & Heilongjiang & Jilin & Jilin Heilongjiang & Neimenggu \\
\hline 0.01 & 10.2 & 11.2 & 10.2 & 13.0 & 6.6 & 11.2 \\
0.02 & 9.8 & 10.6 & 9.8 & 12.4 & 6.4 & 10.8 \\
0.05 & 6.4 & 9.4 & 8.8 & 7.6 & 5.0 & 6.8 \\
0.10 & 5.4 & 8.0 & 3.0 & 6.4 & 3.6 & 5.6 \\
0.20 & 3.2 & 4.8 & 2.0 & 2.4 & 2.6 & 3.0 \\
\hline
\end{tabular}

It can be learned from table 3 that when a flood disaster encountered once in a hundred years happens, almost $10.2 \%$ arable land in Jilin district of the main stream of Songhua River, $11.2 \%$ around arable land in Heilongjiang district of the main stream of Songhua River and about $13.0 \%$ arable land in Jilin district of Nen River will be affected; likewise, when a flood disaster encountered once in five years happens, nearly 3.2\% arable land in Jilin district of the main stream of Songhua River, and almost $4.8 \%$ arable land in Heilongjiang district of the main stream of Songhua 
River will be affected, etc. Concerning disaster-affected rate of arable land, the flood disaster risk lying in Songhua River Basin is relatively low.

\section{Conclusion and Discussion}

In this paper, based on the short time series of flood disaster historic data, the flood disaster risk estimation in Songhua River Basin has been done divisionally through flood disaster loss probability distribution estimation using information diffusion method, which is superior to the traditional statistic model. It is worth to mention that the division for Songhua River Basin in this paper is comparatively cursory, so that the analysis results can not yet completely reflect the spatial differences of flood disaster risk. More accurate division will definitely help to understand the spatial characteristics of flood disaster risk of Songhua River Basin, but the collection of historic disaster data is extremely difficult. In view of the facts that the theoretic system of flood risk evaluation has not been perfect enough so far, and the observed series of flood disaster are quite short or even unavailable, the method based on information diffusion adopted in the paper is indisputably an effective and practical method. However, when it comes to the form and adaptive condition of diffusion function and the determination of diffusion coefficients, further in-depth research should be conducted in the future, in order to ensure the estimation probability values get much closer to the real happening values.

\section{References}

1. Shifeng Huang, Chenghu Zhou, Qing Wan: Primary Analysis on Flood Disaster Risk Evaluation. Geographical Research, Vol. 17. (Supplement) (1998) 71-77

2. Chenghu Zhou, Qing Wan, Shifeng Huang, Deqing Chen: A GIS-based Approach to Flood Risk Zonation. Acta Geographica Sinica, Vol. 55. (2000) 15-24

3. Chongfu Huang and Yong Shi: Towards Efficient Fuzzy Information Processing--Using the Principle of Information Diffusion. Physica-Verlag (Springer), Heidelberg, Germany, (2002)

4. Dianying, Zhu: History of Drought and Flood in the Past 240 Years in Heilongjiang Province. Heilongjiang Technology, Harbin, China (1991) 